International Journal of Biomedicine I June 2019 - Volume 9, Issue Suppl_1: Abstracts From the Second Russian International Conference "Cryo-electron microscopy 2019: achievements and prospects"

POSTER ABSTRACT PRESENTATIONS

SESSION TITLE: STRUCTURE AND FUNCTIONS OF THE TRANSCRIPTION AND TRANSLATION APPARATUS OF THE CELL

DOI: 10.21103/IJBM.9.Suppl_1.P19

\title{
Abstract P-19: Cryo-EM Structure of a New Thiazole/Oxazole-Modified Microcin - Phasolicin - in Complex with Bacterial Ribosome Provides Basis for Species-Specific Activity of Ribosome-Targeting Antibiotics
}

\author{
Dmitrii Y. Travin ${ }^{1,2}$, Zoe Watson ${ }^{3}$, Mikhail Metelev ${ }^{1,2}$, Fred Ward ${ }^{3}$, Ilya A. Osterman ${ }^{1,4}$, \\ Irina M. Khven ${ }^{4,5}$, Marina Serebryakova ${ }^{4}$, Yury S. Polikanov ${ }^{6,7}$
}

${ }^{1}$ Center for Life Sciences, Skolkovo Institute of Science and Technology, Moscow, Russia; ${ }^{2}$ Institute of Gene Biology, Russian Academy of Science, Moscow, Russia; ${ }^{3}$ Department of Molecular and Cell Biology, University of California, Berkeley, USA; ${ }^{4}$ A.N. Belozersky Institute of Physico-Chemical Biology, Lomonosov Moscow State University, Moscow, Russia; ${ }^{5}$ Department of Bioengineering and Bioinformatics, Lomonosov Moscow State University, Moscow, Russia; ${ }^{6}$ Department of Biological Sciences, University of Illinois at Chicago, Chicago, Illinois, USA; ${ }^{7}$ Waksman Institute for Microbiology, Rutgers, the State University of New Jersey, Piscataway, USA

Background: Ribosomally synthetized and posttranslationally modified peptides (RiPPs) form a rapidly expanding class of natural products and serve as a source of new compounds with various biological activities. Linear azole-containing peptides (LAPs) comprise a relatively small heterogeneous subclass of RiPPs that exhibit extremely diverse mechanisms of action sharing many common structural features.

Results: Here we report the discovery of a new LAP biosynthetic gene cluster Pop5 in the genome of Rhizobium sp., which led to the identification of phasolicin (PHS) - an extensively modified ribosomally synthesized peptide exhibiting narrow-spectrum antibacterial activity against a set of bacterial species from Rhizobiales, symbiotic bacteria from the root nodules of various leguminous plants. PHS compound inhibits prokaryotic translation by binding to the $70 \mathrm{~S}$ ribosome and obstructing nascent peptide exit tunnel through which an emerging protein exits the ribosome. We have also obtained cryoEM structure of the Escherichia coli ribosome in complex with PHS that revealed a notably different mode of interaction with both the 23S rRNA and the ribosomal proteins uL4 and uL22 as compared with the recently published LAP klebsazolicin (KLB). Unlike KLB, PHS binds further away from the peptidyl transferase center where it interacts with the loops of proteins uL4 and uL22. Our microbiological data 
suggest that the sequence of the protein uL4 loop determines whether the PHS compound can bind to the ribosome and provides the basis for the species-specific activity of ribosome-targeting antibiotics.

Conclusions: PHS and its predicted homologs from genomes of other bacterial species expand the known diversity of LAPs, which potentially can be used as biocontrol agents for the needs of agriculture.

Key Words: Phasolicin $\bullet$ translation inhibitor $\bullet$ antibiotic $\bullet$ linear azole-containing peptides $\bullet$ RiPPs $\bullet$ Rhizobium natural products

International Journal of Biomedicine. 2019;9 Suppl 1: S25. doi: 10.21103/IJBM.9.Suppl_1.P19

(C)2019 International Medical Research and Development Corporation 